short time after drinking, will produce excessive vomiting. one and bringsit down to 1221 pages. It is expected that this The patient must not know the cause of the vomiting. These volume will be followed with a volume on general pathology things can be worked in the tonic without arousing the in due course.

patient's suspicion; thus the trick of these institutions. The No words of ours are needed in commendation of this maghypodermic is used when a tonic is given hypodermically, on nificent work, without which the library of no pathologist some pretext, after drinking.

Yours fraternally, H. G. NonTon, M.D.

\title{
Medical Laws of the State of Washington.
}

Seattle, Wash., Nov. 10, 1897.

To the Editor:-I am in receipt of many inquiries concern. ing the medical laws of the State of Washington. I would say that all persons intending to practice medicine or surgery in the State of Washington are obliged to pass an examination before the State Board of Medical Examiners.

These examinations are held the first Tuesday in January and the first Tuesday in July. The January meeting is held on the west side of the Cascade mountains and the July meeting on the east side.

No temporary certificates are granted by this Board, and no exception is made to this rule.

F. H. Coe., Sec. Med. Soc. State of Washington.

\section{Correction.}

NEW YoRK, Nov. 8, 1897.

To the Editor:-Please publish the following errata: Joun N L of Nov. 6, 1897, p. 972, second column, lines 21 and 22 , for "medium" please read median; line 32, for "them" read thon; line 43, for "per os" read per orem. I am anxious to have the word thon come into general use, as it is needed very much. Yours truly, E. Cutrer, M.D.

\section{BOOK NOTICES.}

Text-book of Nervous Diseases. By Charles L. Dana, A.M., M.D. New York. Fourth revised edition. One volume, octavo, pp. 640. Profusely illustrated. Muslin, $\$ 3.50$ net. New York: William Wood and Company. 1897.

This edition of the book has been given a very complete revision, and some parts of it have been entirely rewritten. Says the author: "Those who have the pleasure of knowing him personally and his conscientious work will accept his statement as they would his bond." A chapter has been added on alcoholic meningitis, a very necessary addition, and one that should appeal especially to those connected with ambulance service everywhere. Such additions have been made to the therapeutics as the experience of the author has seemed to him to warrant.

The author says: "The number of nervous diseases has not lessened as the years have rolled by, but their grouping and relations are better understood, and the essential unity of many groups has been made more distinct. Those who read the present volume will perhaps be somewhat disturbed at first by the new nomenclature, which has so much to say about neurons, dendrites and neuraxons. These have come to stay, that is, anatomically, and the modern student must become familiar with them. I have to confess, however, that our conception of the neuron has aided us more in our anatomic work than in our pathology. A practical application of the neuron to disease has not yet furnished us very much help."

A Text-book of Special Pathological Anatomy. By ERnest ZiegLer. Translated by Donald MacAllister, M.D., M.A., and Henry W. Cattell, M.D. Sections 9 to 15 . New York and London: The Macmillan Company. 1897.

These sections containing the work as heretofore noticed in the Journal are devoted to the pathology of the liver and pancreas, the respiratory system, the urinary organs, the genital system and the eye and ear.

The paging of the work is continuous with the preceding would be complete.

The style of the translation and the arrangement of the topice resembles the volume of D. J. Hamilton, but the volumes are richer in illustration. At the end of each chapter there is a very full list of references to the literature of the subject treated.

Hare's Practical Diagnosis. The Use of Symptoms in the Diag nosis of Disease. By Hobart Amory Hare, M.D., Professor of Therapeutics and Materia Medica in the Jefferson Medical College of Philadelphia, Laureate of the Medical Society of London, of the Royal Academy in Belgium, etc. New (2d) and revised edition. In one octavo volume of 598 pages with 201 engravings and 13 full-page colored plates. Cloth, \$4.75. Philadelphia: Lea Brothers and Co., Publishers.

In our review of the first edition of this book (see JournaL, Sept. 26, 1896, page 718), we made the following statement:

"We have here a work in which semeiology, the visible symptoms with which we are confronted, is made to tell the story of the patient's pathologic condition, the natural or Hippocratic method. The work is profusely illustrated and the illustra. tions are fine. The title of the volume is not a misnomer in this instance, for it is indeed a practical diagnosis. An excellent index concludes the volume."

There seems to be few changes from the former edition and we have little to add to what we then said.

Clinical Diagnosis. The Bacteriological, Chemical and Microscopical Evidences of Disease. By Dr. RudolpH v. JAKSCH, Translated from the fourth German edition and enlarged by James CAGNex, M.A., M.D. Third edition, with numerous illustrations, partly in colors. Pages, 523: price, \$6. London: Charles Griffin \& Co., Limited. 1897.

This is the third English edition of Professor v. Jaksch's work, which has been largely rewritten and reconstructed. There is no work on the subject more authoritative than this and there are few, if any, more comprehensive of the essentials. Even the most minute are found here and to the practical worker no better guide to clinical diagnosis can be had. The day has gone by when much is left to chance in the matter of diagnosis, as by examination of excrementitious matters, the blood, the urine, histologically and bacteriologically, exact conditions may be understood.

This work is divided into ten chapters, of which Chapter 1 is on the blood; 2 , the buccal secretion; 3 , the nasal secretion; 4 , the sputum; 5 , gastric juice; 6 , the feces; 7 , examination of the urine; 8 , exudations, transudations and cystic fluids ; 9 , secretions of the genital organs, including mammary secretion : 10 , methods of bacteriolngic research.

Simon's Clinical Diagnosis. New (2d) edition, revised and en larged. A Manual of Clinical Diagnosis by Microscopical and Chemical Methods. For students, hospital physicians and practitioners. By Charles E. Simon, M.D., late Assistant Resident Physician Johns Hopkins Hospital, Baltimore. In one very handsome octavo volume of 530 pages, with 135 engravings and 14 full page colored plates. Cloth, $\$ 3.50$.

The present edition of the volume has been brought thoroughly up to date. The parasitology and bacteriology of the blood, saliva, feces, urine and vaginal discharges have been written. New methods of clinical examination, which have appeared since the publication of the first edition, have been included in the work. Numerous additions have been made and the size of the volume increased about fifty pages. The illustrations are excellent and include a number of handsome chromolithographs. The publishers have apparently spared no pains to make the work complete and valuable. The rapid exhaustion of the first edition, published in 1896, indicates the favorable reception of the progress of the work. 